Research

Open Access

\title{
Clinical relevance of the interleukin 10 promoter polymorphisms in Chinese Han patients with major trauma: genetic association studies
}

\author{
Ling Zeng ${ }^{1 *}$, Wei Gu ${ }^{1 *}$, Kehong Chen ${ }^{1}$, Dongpo Jiang ${ }^{2}$, Lianyang Zhang ${ }^{2}$, Dingyuan $\mathrm{Du}^{3}$, Ping $\mathrm{Hu}^{3}$, \\ Qing Liu ${ }^{1}$, Suna Huang ${ }^{1}$ and Jianxin Jiang ${ }^{1}$
}

\author{
1State Key Laboratory of Trauma, Burns and Combined Injury, Research Institute of Surgery, Daping Hospital, Third Military Medical University, \\ Changjiang Road, Yuzhong District, Chongqing, 400042, PR China \\ 2Department of Traumatic Surgery, Daping Hospital, Third Military Medical University, Changjiang Road, Yuzhong District, Chongqing, 400042, PR \\ China \\ ${ }^{3}$ Chongqing Emergency Medical Center, Jiankang Road, Yuzhong District, Chongqing, 400042, PR China \\ * Contributed equally \\ Corresponding author: Jianxin Jiang, hellojjx@126.com
}

Received: 6 Aug 2009 Revisions requested: 28 Sep 2009 Revisions received: 17 Oct 2009 Accepted: 26 Nov 2009 Published: 26 Nov 2009

Critical Care 2009, 13:R188 (doi:10.1186/cc8182)

This article is online at: http://ccforum.com/content/13/6/R188

(c) 2009 Zeng et al; licensee BioMed Central Ltd.

This is an open access article distributed under the terms of the Creative Commons Attribution License (http://creativecommons.org/licenses/by/2.0), which permits unrestricted use, distribution, and reproduction in any medium, provided the original work is properly cited.

\begin{abstract}
Introduction An excessive inflammatory response is thought to account for the pathogenesis of sepsis and multiple organ dysfunction syndrome (MODS) after severe trauma. The interleukin-10 (IL-10) is a potent anti-inflammatory cytokine. The objectives of this prospective study were to investigate the distribution of IL-10 promoter polymorphisms in a cohort of 308 Chinese Han patients with major trauma, and to identify associations of IL-10 promoter polymorphisms with IL-10 production and incidence of sepsis and MODS.
\end{abstract}

Methods A total of 308 patients with major trauma were included in this study. The genotypes of polymorphisms -1082 , -819 and -592 were determined by polymerase chain reactionrestriction fragment length polymorphism. The IL-10 levels in the supernatants were determined with enzyme-linked immunoabsorbent assay.
Results The $-1082 \mathrm{~A}$ and $-592 \mathrm{~A}$ alleles were significantly associated with lower lipopolysaccharide-induced IL-10 production in an allele-dose dependent fashion. There was no significant difference for the -819 polymorphism. Except for the -1082 polymorphism, the -819 and -592 polymorphisms were not significantly associated with sepsis morbidity rate and MOD scores.

Conclusions Our results further confirm the functionality of the IL-10 promoter single nucleotide polymorphisms in relation to IL10 production. They also suggest that individual difference in IL10 production in trauma patients might be at least in part related to genetic variations in the IL-10 promoter region.

\section{Introduction}

Trauma, a major public health problem worldwide, ranks as the fourth leading cause of death among all diseases [1]. One of the most serious complications of major trauma is the sequential dysfunction of vital organs, which is associated with posttraumatic sepsis in the majority of cases [2]. Therefore, preventing sepsis and subsequent organ dysfunction is crucial in the treatment of surviving patients with major trauma. It has been demonstrated that inappropriate immune inflammatory response contributes to the development of sepsis and multiple organ dysfunction syndrome (MODS) in major trauma patients $[3,4]$. Increasing evidence suggests that genetic variants, particularly single nucleotide polymorphisms (SNPs), are critical determinants for inter-individual differences in both inflammatory responses and clinical outcome in trauma patients $[5,6]$. Delineating the variation in genes and associ-

Cl: confidence interval; ELISA: enzyme-linked immunosorbent assay; IFN- $\gamma$ : interferon- $\gamma$; IL-10: interleukin-10; ISS: Injury Severity Score; LPS: lipopolysaccharide; MODS: multiple organ dysfunction syndrome; OR: odds ratios; PCR: polymerase chain reaction; RFLP: restriction fragment length polymorphism; SNPs: single nucleotide polymorphisms; TNF- $\alpha$ : tumor necrosis factor alpha. 
ated differences in response to trauma may contribute to the development of new genetically tailored diagnostic and therapeutic interventions that will improve outcome in the patients with major trauma.

$\mathrm{IL}-10$ is one of the key anti-inflammatory cytokines and it decreases the production of inflammatory molecules, such as TNF- $\alpha$, interferon (IFN)- $\gamma$, IL-12, reactive nitric oxide metabolites, major histocompatibility complex molecules [7] and inhibits antigen-specific cytotoxic T cells [8]. The magnitude of the proinflammatory cytokine response, neutrophil infiltration, and injury were shown to be greater in IL-10(-/)-null mice after visceral ischemia-reperfusion injury. Administration of exogenous IL-10 results in a decrease in neutrophil infiltration in IL-10 (-/-)null mice [9]. It suggests that lower producers of IL-10 might be sensitive to the development of sepsis and MODS due to its ability to suppress inflammation. IL-10 has been shown to be elevated after trauma [10] and is well correlated with the development of sepsis and outcome in patients with major trauma [11,12]. Previous studies have demonstrated that the variation in IL-10 production is largely genetically determined, which is mainly related to genetic variations in the promoter region $[13,14]$. The IL-10 5'-flanking region, which controls transcription, is polymorphic, with two microsatellites between -4000 and -1100 , and three SNPs $(-1082,-819$, and -592) [15]. The $-1082,-819$ and -592 polymorphisms are shown to be in close linkage disequilibrium and construct only three haplotypes in the white population (GCC, ACC and ATA in an order of -1089/-819/-592) [16]. These haplotypes are associated with high (GCC), intermediate (ACC), and low (ATA) IL10 production [14]. Genetic association studies have indicated that the three SNPs in the IL-10 promoter are linked to various diseases, such as Crohn's disease [16], schizophrenia [17], hepatitis [18], endometriosis [19], Alzheimer's disease [20], acute respiratory distress syndrome [21] and sepsis [22]. However, clinical relevance of the three SNPs is still not fully understood, due to considerable controversy in the literature regarding the influence of these SNPs on susceptibility to diseases and their functionality [23-25]. Schroeder and colleagues $[23,26]$ reported the association of the IL-10 with the -1082 and -592 polymorphisms with the incidence of MODS and acute respiratory failure in patients with major trauma, respectively, and also showed inconsistent results.

In this study we investigated whether the genetic variations at positions $-1082,-819$ and -592 in the IL-10 promoter affect IL10 production after trauma, and whether there is an association of these polymorphisms with the development of posttraumatic sepsis and MODS. Our hypothesis was that genetically determined lower production of IL-10 might increase susceptibility to post-traumatic complications.

\section{Materials and methods Study population}

A total of 308 patients with major trauma (240 male and 68 female) were prospectively recruited in this study. All of them are Han Chinese and live in Chongqing district. The patients were consecutively admitted to the Department of Trauma Surgery in the Daping Hospital and the Chongqing Emergency Medical Center between 1 January, 2005 and 1 June, 2008. They were enrolled in the study if they met the following criteria: (1) aged between 18 and 65 years; (2) expected Injury Severity Score (ISS) greater than 16 combined with the presence of at least one life-threatening injury and at least one additional severe injury in another part of the body and (3) probability of survival greater than 48 hours. Patients were not eligible if they had penetrating injuries, or pre-existing cardiovascular, respiratory, renal, hepatic, hematologic or immunological diseases. ISS was performed according to the Abbreviated Injury Scale 2005 by independent evaluators [27]. All patients requiring surgical intervention received standard surgical care and postoperative intensive care unit treatment. The protocol for this study was approved by the Ethical and Protocol Review Committee of the Third Military Medical University, and informed consent was obtained from the patients or the patient's next of kin.

\section{Genotyping of the IL-10 promoter polymorphisms}

Blood specimens were collected in tripotassium ethylenediamine tetraacetic acid sterile tubes from trauma patients immediately after admission (generally within 10 hours after injury) in order to avoid the effect of blood transfusion. The genomic DNA was isolated from whole blood using Wizard genomic DNA purification kit (Promega, Madison, WI, USA) according to the manufacturer's protocol. A PCR-restriction fragment length polymorphism (RFLP) method was used to detect the IL-10 promoter polymorphisms. The oligonucleotide primers for amplification and PCR conditions were shown in Table 1. PCR products were digested with Xagl, Hin $1 \alpha$ or Rsa I (New England Biolabs, Beverly, MA, USA) for one hours at $37^{\circ} \mathrm{C}$. The SNPs were then genotyped by fragment size obtained after agarose gel electrophoresis, which were further confirmed by DNA sequencing of the IL-10 promoter with 10 random samples (Takara Biotech, Dalian, China). The genotyping was performed in a blinded fashion such that those analyzing the genotype data did not know any other experimental results.

\section{Ex vivo IL-10 production}

A human whole-blood assay was used as described previously [6]. In brief, aliquots of whole blood collected from the trauma patients were mixed in a ratio of $1: 1$ with Roosevelt Park Memorial Institute medium 1640, and incubated with $100 \mathrm{ng} /$ $\mathrm{ml}$ lipopolysaccharide (LPS; Escherichia coli O26:B6, Difco Laboratories, Detroit, MI, USA) in a sample mixer at $37^{\circ} \mathrm{C}$ for four hours. The supernatants were then separated by centrifugation. The IL-10 levels in the supernatants were determined 
Table 1

\begin{tabular}{|c|c|c|c|}
\hline SNPs & Primers & PCR conditions & Restriction endonucleases \\
\hline \multirow[t]{2}{*}{$-1082 A / G$} & $\begin{array}{c}\text { F:ACACAAATCCAAGACAACACTACTAA } \\
\text { GGCTTCCTTGGGA }\end{array}$ & $\begin{array}{c}3 \text { minutes at } 94^{\circ} \mathrm{C} \text { followed by } 35 \text { cycles of } 40 \text { seconds } \\
\text { at } 94^{\circ} \mathrm{C}, 45 \text { seconds at } 56^{\circ} \mathrm{C}, 40 \text { seconds at } 72^{\circ} \mathrm{C} \text {, } \\
\text { then } 10 \text { minutes at } 72^{\circ} \mathrm{C}\end{array}$ & Xagl \\
\hline & R:GTGATCAAACAGAGGCACAGACAT & & \\
\hline \multirow[t]{2}{*}{$-819 T / C$} & F:САCTCTAAGGCTTCCTTGGGA & $\begin{array}{c}3 \text { minutes at } 94^{\circ} \mathrm{C} \text { followed by } 35 \text { cycles of } 40 \text { seconds } \\
\text { at } 94^{\circ} \mathrm{C}, 45 \text { seconds at } 56^{\circ} \mathrm{C}, 40 \text { seconds at } 72^{\circ} \mathrm{C} \text {, } \\
\text { then } 10 \text { minutes at } 72^{\circ} \mathrm{C}\end{array}$ & $\operatorname{Hin} 1 \alpha$ \\
\hline & $\begin{array}{c}\text { R:CCTACCGTCTCTATTTTATAGTGAGC } \\
\text { AAACTGAGGCACAGACAT }\end{array}$ & & \\
\hline$-592 A / C$ & F:AGCTGAAGAGGTGGAAACATGTG & $\begin{array}{c}3 \text { minutes at } 94^{\circ} \mathrm{C} \text { followed by } 35 \text { cycles of } 40 \text { seconds } \\
\text { at } 94^{\circ} \mathrm{C}, 45 \text { seconds at } 63^{\circ} \mathrm{C}, 40 \text { seconds at } 72^{\circ} \mathrm{C} \text {, } \\
\text { then } 10 \text { minutes at } 72^{\circ} \mathrm{C}\end{array}$ & Rsa I \\
\hline
\end{tabular}

by ELISA according to the manufacturer's instructions ( $R$ \& $D$ System, Minneapolis, MN, USA). The lower limit for detection was $4 \mathrm{pg} / \mathrm{ml}$. Variability between triplicate wells was less than $5 \%$.

\section{Clinical evaluation}

After admission, the patients with major trauma were monitored in the following aspects: respiratory (partial pressure of arterial oxygen/fraction of inspired oxygen ratio), renal (serum creatinine concentration), hepatic (serum bilirubin concentration), cardiovascular (pressure-adjusted heart rate) and hematologic (platelet count) systems. The organ function was then scored using the method of Marshall and colleagues [28] and calculated as a single daily value during the intensive care unit stay. Neurological scoring was not performed because every patient was sedated. Sepsis was defined if patients met all the following criteria: clinical evidence of infection, body temperature greater than $38.5^{\circ} \mathrm{C}$ or less than $36.5^{\circ} \mathrm{C}$, and leukocyte count greater than $10 \times 10^{9} / \mathrm{L}$ or less than $4 \times 10^{9} / \mathrm{L}$. The MODS scores and sepsis were determined by individuals who did not know the genotypes.

\section{Statistical analysis}

Sample size was calculated using online Power and Sample Size Program software [29]. The desired power of our study was set at $80 \%$ with a significance level of 0.05 in a two-sided test. We chose the log-additive inheritance model, which is the most suitable for polygenic diseases. By means of the Power and Sample Size program, our sample $(n=308)$ was considered adequate to study the $-1082,-819$ and -592 polymorphisms.

Allele frequencies for each SNP were determined by gene counting. Any deviation from Hardy-Weinberg equilibrium was calculated by a chi-squared goodness-of-fit test. The chi- squared test or Fisher's exact test was used to determine differences in frequencies of the IL-10 promoter polymorphisms among trauma patients with different genotypes. Odds ratios $(\mathrm{OR})$ and $95 \%$ confidence intervals $(\mathrm{Cl})$ were calculated using logistic regression models whenever that chi-squared or Fisher's exact test was significant. The association of the IL-10 promoter polymorphisms with plasma IL-10 levels and MODS scores was determined using one-way analysis of variance. Three genetic models (allele-dose, dominant, and recessive) were used. Significant probability values obtained were corrected for multiple testing (Bonferroni correction). $P$ values less than 0.05 were considered significant. All statistic analysis was carried out using SPSS Version 11.0 (SPSS Inc, Chicago, Illinois, USA).

\section{Results \\ Overall clinical characteristics of patients with major trauma}

The patient cohort comprised a total of 308 consecutive Han Chinese patients with severe multiple trauma. Trauma was caused by traffic accidents ( $n=187)$, falling ( $n=105)$, or other causes $(n=16)$. Baseline data of the patients are shown in Table 2. The severely injured patients (mean ISS $25.5 \pm 8.2$ ) were mostly young (38.5 \pm 11.5 years). One hundred and forty seven patients (47.7\%) developed sepsis. Two hundred and fifty five $(82.8 \%)$ patients developed organ dysfunction, among whom one hundred and sixty (52\%) had two or more organ dysfunctions. The mean time to MODS was $4.4 \pm 3.5$ days. The mean number of operations performed per patient was $3.5 \pm 3.2$ (range 1 to 14). Substitution of erythrocytes was necessary in every patient (mean $5.4 \pm 5.2 \mathrm{~L}$ ). All of the patients survived at least 48 hours after admission and completed genotyping. 
Table 2

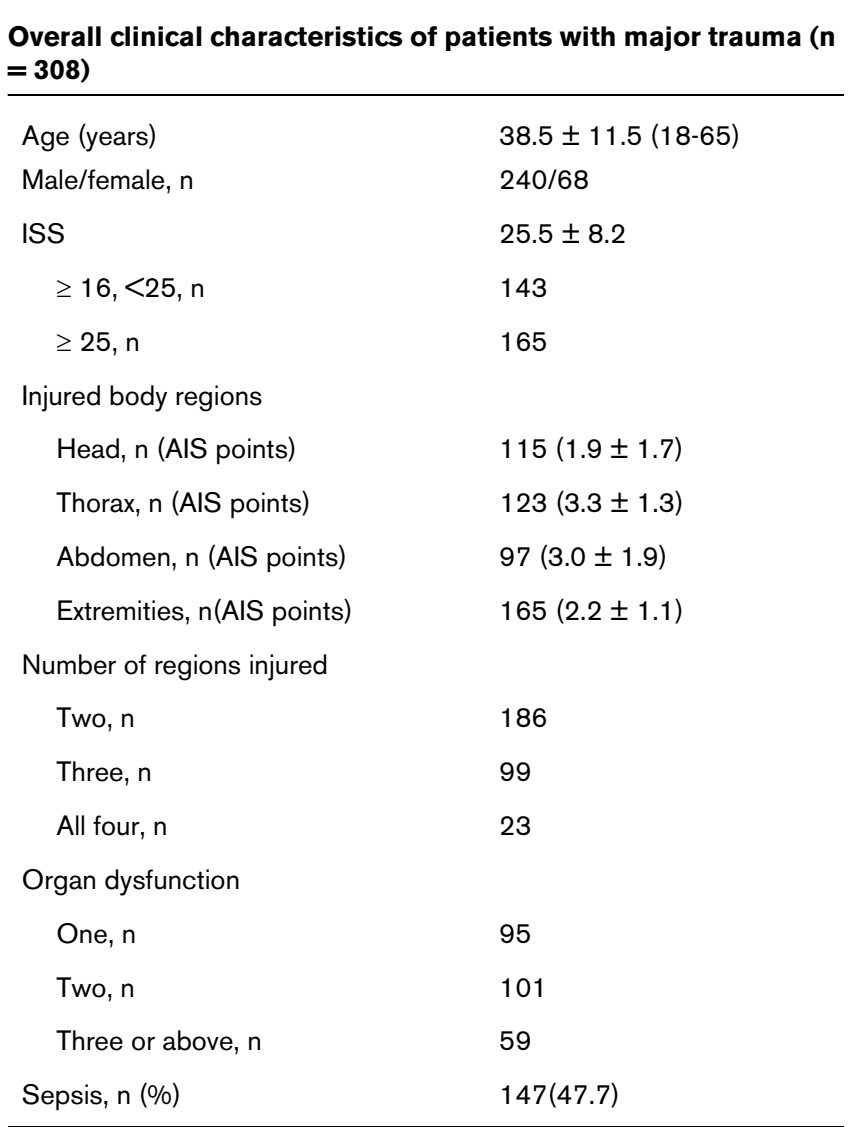

AIS = Abbreviated Injury Scale; ISS = Injury Severity Scores.

\section{Allele frequencies and genotype distribution}

The overall minor allele frequencies were $15.4 \%, 29.2 \%$ and $34 \%$ for the $-1082 \mathrm{G},-819 \mathrm{C}$ and $-592 \mathrm{C}$ alleles, respectively, in our cohort. The genotype frequencies of these three SNPs were in agreement with the Hardy-Weinberg equilibrium (Table 3). These SNPs were completely $(-819$ to -592$)$ or strongly (-1082 to -819 and -592$)$ linked. The three common haplotypes with frequency of more than or equal to $10 \%$ were $-1082 \mathrm{~A} /-819 \mathrm{~T} /-592 \mathrm{~A}$ (ATA, 49.5\%), -1082A/-819T/-592C (ATC, 14.1\%) and -1082A/-819C/-592A (ACA, 13.1\%), respectively. With regard to the number of ATA haplotypes, $25.7 \%$ of the trauma patients had the genotype 0 ATA, $51.2 \%$ had 1 ATA, and $23.1 \%$ had 2 ATA.

\section{Association of the IL-10 promoter polymorphisms with IL-10 production}

Given the possible functionality of the $-1082,-819$ and -592 polymorphisms, we hypothesized that these SNPs might affect IL-10 production after trauma, leading to individual difference in plasma IL-10 levels in trauma patients. Peripheral blood was taken immediately after admission in an attempt to avoid the potential effects of non-genetic factors on IL-10 production. As shown in Table 4, there were no significant differences in age, gender ratio and ISS scores among trauma patients with different genotypes of the three polymorphisms. Spontaneous IL-10 production was not significantly different between different genotypes of all three loci (data not shown). However, LPS-induced IL-10 production was shown to be significantly different between different genotypes of the -1082 and -592 polymorphisms, showing that the $-1082 \mathrm{~A}$ and $-592 \mathrm{~A}$ alleles were associated with lower $\mathrm{IL}-10$ production $(P=$ 0.005 and $P=0.001$, respectively by dominant effect). Data from linear regression analysis indicated that the functional association of these two polymorphisms with IL-10 production was significantly allele-dose dependent $(P=0.003$ and $P=$

Table 3

Distribution of the IL-10 promoter polymorphisms among trauma patients

\begin{tabular}{|c|c|c|c|c|c|c|c|}
\hline \multirow[t]{2}{*}{ Loci } & \multirow[t]{2}{*}{ Patients } & \multirow[t]{2}{*}{$\mathrm{n}$} & \multirow[t]{2}{*}{ MA (\%) } & \multicolumn{3}{|c|}{ Genotypes, n (\%) } & \multirow[t]{2}{*}{ HWE } \\
\hline & & & & wild & heterozygous & variant & \\
\hline \multirow[t]{3}{*}{$A-1082 G$} & All & 308 & 15.4 & $224(72.7)$ & $73(23.7)$ & $11(3.6)$ & 0.11 \\
\hline & Sepsis & 147 & 12.2 & $115(78.2)$ & $28(19.1)$ & $4(2.7)$ & \\
\hline & Non-sepsis & 161 & $18.3^{a}$ & $109(67.7)^{b}$ & $45(28)$ & $7(4.3)$ & \\
\hline \multirow[t]{3}{*}{$\mathrm{T}-819 \mathrm{C}$} & All & 308 & 29.2 & $157(51.0)$ & $122(39.6)$ & $29(9.4)$ & 0.46 \\
\hline & Sepsis & 147 & 29.3 & $73(49.7)$ & $62(42.2)$ & $12(8.2)$ & \\
\hline & Non-sepsis & 161 & 29.2 & $84(52.2)$ & 60 (37.3) & $17(10.6)$ & \\
\hline \multirow[t]{3}{*}{ A-592C } & All & 307 & 34.0 & $141(45.9)$ & $123(40.1)$ & $43(14.0)$ & 0.07 \\
\hline & Sepsis & 147 & 34.4 & $65(44.2)$ & $63(42.9)$ & 19 (12.9) & \\
\hline & Non-sepsis & 160 & 33.8 & $76(47.5)$ & $60(37.5)$ & $24(15.0)$ & \\
\hline
\end{tabular}

Sepsis vs. non-sepsis: ${ }^{\mathrm{a} P}=0.037, \mathrm{~b} P=0.038$. $\mathrm{HWE}=$ Hardy-Weinberg equilibrium; $\mathrm{MA}=$ minor allele. 
Table 4

\begin{tabular}{|c|c|c|c|c|c|c|c|}
\hline Genotypes & $\mathbf{N}$ & Age (years) & $\begin{array}{c}\text { Gender } \\
(\mathrm{M} / \mathrm{F})\end{array}$ & ISS & Sepsis(\%) & MOD score & Cytokine (pg/ml) \\
\hline \multicolumn{8}{|l|}{-1082} \\
\hline AA & 224 & $38.6 \pm 14.1$ & $177 / 47$ & $25.6 \pm 8.5$ & 51.3 & $4.4 \pm 2.7$ & $62.0 \pm 19.8$ \\
\hline$A G$ & 73 & $37.3 \pm 13.5$ & $62 / 11$ & $26.3 \pm 7.5$ & 38.4 & $5.4 \pm 2.8$ & $73.9 \pm 21.3$ \\
\hline GG & 11 & $35.9 \pm 20.3$ & $7 / 4$ & $27.2 \pm 10.6$ & 36.4 & $4.1 \pm 2.9$ & $86.0 \pm 18.8$ \\
\hline-819 & & & & & a1 & b1 & $\mathrm{a} 2, \mathrm{~b} 2, \mathrm{c} 1$ \\
\hline $\mathrm{TT}$ & 157 & $37.6 \pm 13.7$ & $120 / 37$ & $25.6 \pm 8.8$ & 46.5 & $4.6 \pm 2.6$ & $56.2 \pm 17.8$ \\
\hline $\mathrm{TC}$ & 122 & $39.6 \pm 14.6$ & $100 / 22$ & $25.9 \pm 8.1$ & 50.8 & $4.7 \pm 2.8$ & $59.9 \pm 20.2$ \\
\hline $\mathrm{CC}$ & 29 & $35.7 \pm 15.1$ & $26 / 3$ & $27.1 \pm 7.9$ & 41.4 & $4.4 \pm 2.9$ & $62.4 \pm 19.5$ \\
\hline \multicolumn{8}{|l|}{-592} \\
\hline AA & 141 & $38.2 \pm 14.6$ & $116 / 25$ & $25.1 \pm 7.9$ & 46.1 & $4.5 \pm 2.1$ & $52.9 \pm 16.0$ \\
\hline$A C$ & 123 & $38.0 \pm 13.2$ & $98 / 25$ & $27.1 \pm 9.0$ & 51.2 & $5.1 \pm 3.0$ & $66.8 \pm 19.6$ \\
\hline \multirow[t]{2}{*}{$\mathrm{CC}$} & 43 & $36.8 \pm 16.0$ & $31 / 12$ & $24.8 \pm 8.0$ & 44.2 & $4.1 \pm 2.7$ & $59.8 \pm 13.1$ \\
\hline & & & & & & & a3, c2 \\
\hline
\end{tabular}

a: dominant effect (variant homozygotes theterozygotes vs. wild homozygotes) as analyzed by ANCOVA, a1 $P=0.038$, a2 $P=0.005$, a3 $P=0.001$. b: recessive effect (variant homozygotes vs. heterozygotes + wild homozygotes) as analyzed by ANCOVA, ${ }^{b} 1 P=0.088,{ }^{b 2} P=0.083$. c: allele dose effect as analyzed by linear regression analysis, ${ }^{c 1} P=0.003,{ }^{c 2} P=0.037$.

ANCOVA = analysis of covariance; $F=$ female; ISS = Injury Severity Score; $M=$ male; MOD = multiple organ dysfunction.

0.037 for -1082 and -592 , respectively). Although the $-819 \mathrm{~T}$ allele is also associated with lower LPS-induced IL-10 production, no significant difference was found between the different genotype groups (Table 4). In case of a combination effect of the three SNPs, we further analyzed the association of ATA haplotype with IL-10 production. Table 5 showed that the production of IL-10 was lowest in those with haplotype genotype 2 ATA, showing significant difference if compared with those with genotype 0 ATA $(P=0.041)$. Data from linear regression analysis indicated that this association with IL-10 production were significantly haplotype-dose dependent $(P=0.041)$.

\section{Association of the IL-10 promoter polymorphisms with development of sepsis and MODS in trauma patients}

As shown in Table 4, there was a close association of the 1082 polymorphism with the development of sepsis, showing that the patients with the A allele had significantly higher sepsis morbidity $(P=0.038$ for dominant effect). Data from multiple logistical regression analyses indicated that $A$ to $G$ variation at this position was borderline significantly associated with lower risk of sepsis (OR $=0.677,95 \% \mathrm{Cl}=0.453$ to $1.011, P=0.057)$. In addition, there was a borderline significantly increasing trend of MODS scores in the patients with the $\mathrm{A}$ allele at -1082 locus ( $P=0.088$ for recessive effect). However, both of the -819 and -592 polymorphisms were not

Table 5

\begin{tabular}{ccccc}
\hline \multicolumn{2}{l}{ Clinical relevance of Interleukin-10 haplotypes in major trauma patients } \\
\hline Haplotype $^{*}$ & Count & IL-10 $(\mathbf{p g} / \mathbf{m l})$ & MODS & Sepsis (\%) \\
\hline (ATA) & 71 & $72.18 \pm 22.51$ & $4.25 \pm 2.60$ & $28(39.4)$ \\
1(ATA) & 157 & $65.19 \pm 20.36$ & $5.00 \pm 2.89$ & $76(48.4)$ \\
2(ATA) & 79 & $60.27 \pm 19.24^{a, c}$ & $4.24 \pm 2.39$ & $43(54.4)$
\end{tabular}

*'0 ATA haplotype' includes the following genotypes: ATC/ATC, ATC/ACC, ACA/ACA, ACA/ACC, ACC/ACC, ATC/GTC, ATC/GCC, ACC/ GTC, ACA/GCA, ACC/GCA, ACA/GCC, ACC/GCC, GTA/GTC, GTC/GTC, GTA/GCC and GTC/GCA; '1 ATA haplotype' includes ATA/ATC ATA/ACA, ATA/ACC, ATA/GTA, ATA/GTC, ATA/GCA and ATA/GCC; '2 ATA haplotype' is ATA/ATA. haplotype is indicated in an order of 1082A, -819T and -592A.

a: 2 ATA vs 0 ATA, $P=0.041$; c: haplotype dose effect, $P=0.041$.

MODS = multiple-organ dysfunction syndrome. 
shown to be significantly associated with the development of sepsis and MODS in trauma patients.

Concerning the association of sepsis with allele and genotype frequencies, there were no significant differences in allele and genotype frequencies of the three polymorphic loci between all trauma patients and trauma patients with sepsis. There was a significant difference in the AA genotype frequency $(P=$ 0.038 ) at position -1082 between patients with and without sepsis. The frequency of the $\mathrm{G}$ allele was significantly higher in patients without sepsis than in those with sepsis $(P=$ 0.037). No significant difference was observed between patients with and without sepsis for the allele and genotype frequencies of other two polymorphic loci (Table 3). With respect to the combination effect of the three SNPs, although there was a trend of increasing risk for sepsis incidence with the number of ATA haplotype (39.4\% for 0 ATA vs $54.4 \%$ for 2 ATA), no significant differences in MODS scores and sepsis morbidity rate were observed between any haplotype groups (Table 5).

\section{Discussion}

The aim of the present study was to estimate the clinical relevance of the IL-10 promoter polymorphisms in patients with major trauma. The described genotype and allele frequencies of the IL-10 polymorphisms studied in this cohort are quite similar to previously reported distributions in other studies in Chinese patients [18-20,25] and other Asian populations [30]. The minor alleles at positions -819 and -592 are the $C$ allele in Asian populations [18-20,25,30], which is different to those seen in Western populations, being $T$ and $A$ alleles, respectively [22-24,26,31]. Although the minor allele at position -1082 is $\mathrm{G}$ in both Asian and Western populations, its frequency is quite different, over $40 \%$ in Western populations $[21,22,26,31,32]$ and about $10 \%$ in Asian populations [1921]. This leads to quite a difference in the common haplotypes between Asian and Western populations. Three common haplotypes in this cohort are ATA, ATC and ACA, respectively, while GCC, ATA and ACC are the common haplotypes in Western populations. The differences in allele prevalence might be due to ethnic difference [33].

It has been shown that about $75 \%$ of the variation in IL-10 production is genetically determined [13]. IL-10 production appears to be controlled at the transcriptional level [34]. The 1082, -819 and -592 polymorphisms have been shown to affect promoter activity, showing that the ATA haplotype is associated with the lowest transcription activity [19]. Results from bioinformatics analysis indicates that base pair substitutions at positions $-1082,-819$ and -592 might affect putative transcription factor binding, such as C/EBP beta, GATA-binding factor 2, Ets and STAT3 [35]. Given the functionality of the IL-10 promoter SNPs, we investigate whether these SNPs affect IL-10 production in response to a known stimulus such as LPS immediately after trauma. In the case of the effect of individual SNP, our results indicate that both of the $-1082 \mathrm{~A}$ and -592 A alleles are shown to be associated with LPSinduced IL-10 production. However, there is no association for the -819 polymorphism. Our results are in agreement with previous reports [15,34], but in contrast to the results reported by Keijsers and colleagues [36] and Rad and colleagues [37]. They showed that the A allele at -1082 and the T allele at -819 were associated with higher and lower IL-10 production, respectively. Similar to their effect on transcription, LPS-stimulating production of IL-10 by peripheral blood leukocytes is lowest in patients with combined genotype 2 ATA, which is significantly different compared with patients with genotype 0 ATA. The ATA haplotype has been demonstrated to have lowest transcriptional activity [19]. Our results further confirm the functionality of the IL-10 promoter SNPs in relation to IL-10 production. It also suggests that individual differences in IL-10 production in trauma patients might be at least in part related to genetic variations in the $\mathrm{IL}-10$ promoter region.

Given the functional significance of the -1082 and -592 polymorphisms and ATA haplotype, we further hypothesized that the IL-10 promoter polymorphisms would be important in influencing severity to the development of sepsis and MODS in trauma patients by changing the balance of pro-inflammatory and anti-inflammatory cytokines. There were no significant differences in age, gender ratio and ISS scores among the different genotype groups, which minimized the influence of interfering factors. The patient cohort consisted of young patients with a low background of coexisting morbidity. Similar to their association with IL-10 production, genetic variations at the three loci have a trend to be associated with lower risk of sepsis and MODS. However, only the -1082 polymorphism has statistical significance, showing that the patients with the A allele had significantly higher sepsis morbidity and a borderline significant increase in MODS scores. There are also significant differences in the frequencies of the allele and $A A$ genotype at this polymorphic locus between patients with sepsis and non-sepsis. The finding of no significant difference in the GG genotype frequency might be due to a small number of patients carrying the GG genotype in the current study. Results from Stanilova and colleagues [22] also revealed that the AA genotype of the -1082 locus was associated with lower IL-10 production in LPS-, phytohemagglutinin- or pokeweed mitogen-stimulated healthy peripheral blood leukocytes. Patients with severe sepsis were shown to have a significant elevation of $A$ allele frequency when compared with healthy controls. In addition, Gong and colleagues [21] further reported that the high IL-10-producing -1082 GG genotype was protective against organ failure and mortality in acute respiratory distress syndrome. It is increasingly recognized that the overall balance between pro-inflammatory and anti-inflammatory responses is important in the development of sepsis and MODS after trauma. Therefore, it is reasonable that lower IL-10-producing -1082 AA genotype might be used as a relevant risk estimate for organ dysfunction and sepsis in trauma 
patients. This is not consistent with the report by Schroeder and colleagues, which showed that the -592 polymorphism, but not -1082 , was associated with MODS in patients with major trauma [23]. The different patient populations studied may explain this discrepancy. With respect to the combination effect, our results show that the patients with 2 ATA haplotypes have higher sepsis morbidity rates, but this lacks statistical significance, although it is significantly associated with lower IL-10 production. This is in accordance with previous reports [38]. It suggests that there is a lack of synergistic effect among the $-1082,-819$ and -592 polymorphisms in relation to the development of sepsis and MODS in trauma patients. Other pathogenic factors should be also considered when explaining the current results. One important factor is the polygenetic and multifactorial involvement in the pathogenesis of sepsis and multiple organ dysfunction after trauma [39]. In fact, there is increasing evidence indicating that genetic polymorphisms of other genes are associated with the occurrence of post-traumatic complications, such as IL-1 $\beta$, $\mathrm{TNF} \alpha$, heat shock protein $70, \mathrm{IFN}-\gamma$ and IL-18 $[40,41]$. The susceptibility to sepsis and organ dysfunction in trauma patients might be the result of a combination of numerous genetic polymorphisms. In addition, the relatively small sample size we recruited in this study might also affect our conclusions. Our sample, although being considered adequate by means of the Power and Sample Size program, just has a size of about 300 patients, among which only 147 and 160 patients had sepsis and MODS, respectively. Further studies are needed to confirm the clinical relevance of the IL-10 promoter polymorphisms in a larger cohort of trauma patients and to investigate the relation of these SNPs with other genetic polymorphisms in prediction of sepsis and outcome in trauma patients.

\section{Conclusions}

The present study investigated the clinical relevance of the genetic variations at positions $-1082,-819$ and -592 in the IL10 promoter in patients with major trauma. These genetic variations are shown to affect IL-10 production after trauma, and to be associated with risk for the development of post-traumatic sepsis and MODS at different degrees. Further studies, both clinical and experimental, are therefore needed to confirm the significance of these findings and to investigate their synergistic effect with other genetic polymorphisms in relation to the development of sepsis and outcome in trauma patients.

\section{Competing interests}

The authors declare that they have no competing interests.

\section{Authors' contributions}

$L Z$ and $W G$ were the main researchers for this study and cocontributed to write this manuscript. K-HC, D-PJ, L-YZ, D-YD and $\mathrm{PH}$ were involved in the collecting of blood samples and clinical data. $\mathrm{QL}$ and $\mathrm{S}-\mathrm{NH}$ did the technical work. J-XJ planned the study, wrote the protocol and was involved in the

\section{Key messages}

- The -1082A and -592A alleles and ATA haplotype were significantly associated with lower IL-10 production in response to ex vivo LPS stimulation.

- The -1082 polymorphism was closely associated with the development of sepsis, showing that the patients with the A allele had significantly higher sepsis morbidity.

- There are no marked synergistic effects among the $1082,-819$ and -592 polymorphisms in relation to the development of sepsis and MODS in trauma patients.

genetic and clinical aspects of data analyses and revised the manuscript. All authors read and approved the final manuscript.

\section{Acknowledgements}

The authors thank the staff of the department of traumatic Surgery of Daping Hospital and Chongqing Emergency Medical Center for their clinical help and support. The present study was supported by the Major State Basic Research Development Program of China (No. 2005CB522602), and the National Funds for Outstanding Youth Scientists (30325040).

\section{References}

1. Wang ZG, Jiang JX: An overview of research advances in road traffic trauma in China. Traffic Injury Prevention 2003, 4:9-16.

2. Jiang JX: Posttraumatic stress and immune dissonance. Chin J Traumatol 2008, 11:203-208.

3. Lausevic Z, Ausevic ML, Trbojevic-Stankovic J, Krstic S, Stojimirovic $B$ : Predicting multiple organ failure in patients with severe trauma. Can J Surg 2008, 51:97-102.

4. Lenz A, Franklin GA, Cheadle WG: Systemic inflammation after trauma. Injury 2007, 38:1336-1345.

5. Jiang JX: Genomic polymorphisms and sepsis. Chin J Trauma tol 2005, 21:45-49.

6. Gu W, Du DY, Huang J, Zhang LY, Liu Q, Zhu PF, Wang ZG, Jiang $J X$ : Functional significance of gene polymorphisms in the promoter of myeloid differentiation-2. Ann Surg 2007, 246:151-158.

7. Mosmann TR: Properties and functions of interleukin-10. Adv Immunol 1994, 56:1-26.

8. Mosser DM, Zhang X: Interleukin-10: new perspectives on an old cytokine. Immunol Rev 2008, 226:205-218.

9. Welborn Mr, Moldawer LL, Seeger JM, Minter RM, Huber TS: Role of endogenous interleukin-10 in local and distant organ injury after visceral ischemia-reperfusion. Shock 2003, 20:35-40.

10. Neidhardt R, Keel M, Steckholzer U, Safret A, Ungethuem U, Trentz $\mathrm{O}$, Ertel W: Relationship of interleukin-10 plasma levels to severity of injury and clinical outcome in injured patients. $J$ Trauma 1997, 42:863-871.

11. Maier B, Lefering R, Lehnert M, Laurer HL, Steudel WI, Neugebauer EA, Marzi I: Early versus late onset of multiple organ failure is associated with differing patterns of plasma cytokine biomarker expression and outcome after severe trauma. Shock 2007, 28:668-674.

12. McDaniel DO, Hamilton J, Brock M, May W, Calcote L, Tee LY, Vick L, Newman DB, Vick K, Harrison S, Timberlake G, Toevs C: Molecular analysis of inflammatory markers in trauma patients at risk of postinjury complications. J Trauma 2007, 63:147-157.

13. Westendorp RG, Langermans JA, Huizinga TW, Elouali AH, Verweij $\mathrm{CL}$, Boomsma DI, Vandenbroucke JP: Genetic influence on cytokine production and fatal meningococcal disease. Lancet 1997, 349:170-173. 
14. Stewart D, Fulton WB, Wilson C, Monitto CL, Paidas CN, Reeves $\mathrm{RH}$, De Maio A: Genetic contribution to the septic response in a mouse model. Shock 2002, 18:342-347.

15. Turner DM, Williams DM, Sankaran D, Lazarus M, Sinnott PJ, Hutchinson IV: An investigation of polymorphism in the interleukin-10 gene promoter. Eur J Immunogenet 1997, 24:1-8.

16. Fowler EV, Eri R, Hume G, Johnstone S, Pandeya N, Lincoln D, Templeton D, Radford-Smith GL: TNF alpha and IL10 SNPs act together to predict disease behavior in Crohn's disease. J Med Genet 2005, 42:523-528.

17. He G, Zhang J, Li XW, Chen WY, Pan YX, Yang FP, Gu NF, Feng GY, Yang SL, He JY, Liu BX, Peng YW, Liu J, He L: Interleukin10-1082 promoter polymorphism is associated with schizophrenia in a Han Chinese sib-pair study. Neurosci Lett 2006, 394:1-4.

18. Wang J, Ni H, Chen L, Song WQ: Interleukin-10 promoter polymorphism in patients with hepatitis $B$ virus infection or hepatocellular carcinomain Chinese Han ethnic population. Hepatobiliary Pancreat Dis Int 2005, 5:60-64.

19. Zhang K, Hei $P$, Deng L, Lin J: Interleukin-10 gene promoter polymorphisms and their protein production in peritoneal fluid in patients with endometriosis. Mol Hum Reprod 2007, 13:135-140.

20. Ma SL, Tang NL, Lam LC, Chiu HF: The association between promoter polymorphism of the interleukin-10 gene and Alzheimer's disease. Neurobiol Aging 2005, 26:1005-1010.

21. Gong MN, Thompson BT, Williams PL, Zhou W, Wang MZ, Pothier L, Christiani DC: Interleukin-10 polymorphism in position 1082 and acute respiratory distress syndrome. Eur Respir J 2006, 27:674-681.

22. Stanilova SA, Miteva LD, Karakolev ZT, Stefanov CS: Interleukin10-1082 promoter polymorphism in association with cytokine production and sepsis susceptibility. Intensive Care Med 2006, 32:260-266.

23. Schroeder O, Laun RA, Held B, Ekkernkamp A, Schulte KM: Association of interleukin-10 promoter polymorphism with the incidence of multiple organ dysfunction following major trauma: results of a prospective pilot study. Shock 2004, 21:306-310.

24. Romero JM, Lopez PS, Cozar JM, Carretero R, Canton J, Vazquez F, Concha A, Tallada M, Garrido F, Ruiz-Cabello F: A polymorphism in the interleukin-10 promoter affects the course of disease in patients with clear-cell renal carcinoma. Hum Immunol 2009, 70:60-64.

25. Wu F, Qu YB, Tang YX, Cao DZ, Sun P, Xia ZL: Lack of association between cytokine gene polymorphisms and silicosis and pulmonary tuberculosis in Chinese iron miners. J Occup Health 2008, 50:445-454.

26. Schroeder O, Schulte KM, Schroeder J, Ekkernkamp A, Laun RA: The -1082 interleukin-10 polymorphism is associated with acute respiratory failure after major trauma: a prospective cohort study. Surgery 2008, 143:233-242.

27. The Abbreviated Injury Scale. Barrington. Illinois: Association for the Advancement of Automotive Medicine. 2005.

28. Marshall JC Cook DJ, Christou NV, Bernard GR, Sprung CL, Sibbald WJ: Multiple organ dysfunction score: A reliable descriptor of a complex clinical outcome. Crit Care Med 1995, 23:1638-1652.

29. Power and Sample Size Program [http://biostat.mc.vander bilt.edu/twiki/bin/view/Main/PowerSampleSize

30. Chung BS, Lee JK, Choi MH, Park MH, Choi D, Hong ST: Single nucleotide polymorphisms of cytokine genes are associated with fibrosis of the intrahepatic bile duct wall in human clonorchiasis. Korean J Parasitol 2009, 47:145-151.

31. Tseng LH, Storer B, Petersdorf E, Lin MT, Chien JW, Grogan BM, Malkki M, Chen PJ, Zhao LP, Martin PJ, Hansen JA: IL10 and IL10 receptor gene variation and outcomes after unrelated and related hematopoietic cell transplantation. Transplantation 2009, 87:704-710.

32. Pachkoria K, Lucena MI, Crespo E, Ruiz-Cabello F, Lopez-Ortega S, Fernandez M, Romero-Gomez M, Madrazo A, Duran JA, De Dios AM, Borraz Y, Navarro JM, Andrade RJ: Analysis of IL-10, IL-4 and TNF-a polymorphisms in drug-induced liver injury (DILI) and its outcome. J Hepatol 2008, 49:107-114.

33. Colhoun HM, McKeigue PM, Davey Smith G: Problems of reporting genetic associations with complex outcomes. Lancet 2003, 361:865-872.
34. Westendrop RG, Langermans JA, Huizinga TW, Elouali AH, Verweij CL, Boomsma DI, Vandenbroucke JP: Genetic influence of cytokine production and fatal meningococcal disease. Lancet 1997, 349:170-173.

35. MOTIF Search [http://motif.genome.jp]

36. Keijsers V, Verweij CL, Westendorp RGJ, Breedveld FC, Huizinga TWJ: IL10 polymorphisms in relation to production and rheumatoid arthritis. Arthritis Rheum 1997, 40:179-185.

37. Rad R, Dossumbekova A, Neu B, Lang R, Bauer S, Saur D, Gerhard $\mathrm{M}$, Prinz $\mathrm{C}$ : Cytokine gene polymorphism influence mucosal cytokine expression, gastric inflammation and host specific colonization during Helicobacter pylori infection. Gut 2004, 53:1082-1089.

38. Reid CL, Perrey C, Pravica V, Hutchinson IV, Campbell IT: Genetic variation in proinflammatory and anti-inflammatory cytokine production in multiple organ dysfunction syndrome. Crit Care Med 2003, 30:2216-2221.

39. Giannoudis PV, van Griensven M, Tsiridis E, Pape HC: The genetic predisposition to adverse outcome after trauma. J Bone Joint Surg Br 2007, 89:1273-1279.

40. Wen AQ, Wang J, Feng K, Zhu PF, Wang ZG, Jiang JX: Effects of haplotypes in the interleukin 1 beta promoter on lipopolysacchride-induced interleukin 1 beta expression. Shock 2006, 26:25-30.

41. Hildebrand F, Pape HC, van Griensven M, Meier S, Hasenkamp S, Krettek C, Stuhrmann M: Genentic predisposition for a compromised immune system after multiple trauma. Shock 2005 24:518-522 